The University of Maine

DigitalCommons@UMaine

Psychology Faculty Scholarship

Psychology

$10-16-2013$

\title{
Is Pressure Stressful? The Impact of Pressure on the Stress Response and Category Learning
}

Shannon L. McCoy

University of Maine, shannon.mccoy@umit.maine.edu

Steven B. Hutchinson

University of Maine, steven.hutchinson@umit.maine.edu

Lauren Hawthorne

University of Maine, lauren.hawthorne@umit.maine.edu

Brandon J. Cosley

University of South Carolina - Beaufort, brandon.cosley@umit.maine.edu

Shawn W. Ell

University of Maine, shawn.ell@maine.edu

Follow this and additional works at: https://digitalcommons.library.umaine.edu/psy_facpub

Part of the Cognitive Neuroscience Commons, and the Cognitive Psychology Commons

\section{Repository Citation}

McCoy, Shannon L.; Hutchinson, Steven B.; Hawthorne, Lauren; Cosley, Brandon J.; and Ell, Shawn W., "Is Pressure Stressful? The Impact of Pressure on the Stress Response and Category Learning" (2013). Psychology Faculty Scholarship. 25.

https://digitalcommons.library.umaine.edu/psy_facpub/25

This Article is brought to you for free and open access by DigitalCommons@UMaine. It has been accepted for inclusion in Psychology Faculty Scholarship by an authorized administrator of DigitalCommons@UMaine. For more information, please contact

um.library.technical.services@maine.edu. 


\title{
Is Pressure Stressful? The Impact of Pressure on the Stress Response and Category Learning
}

\author{
Shannon K. McCoy ${ }^{1}$, Steven Hutchinson ${ }^{1}$, Lauren Hawthorne ${ }^{1}$, Brandon J. Cosley ${ }^{2}$, Shawn W. Ell ${ }^{1,3}$ \\ ${ }_{1}^{1}$ Psychology Department, University of Maine, Orono, Maine \\ ${ }^{2}$ Department of Social Science, University of South Carolina, Beaufort, Blufton, South Carolina \\ ${ }^{3}$ Graduate School of Biomedical Sciences, University of Maine, Orono, Maine
}

\begin{abstract}
We examine the basic question of whether pressure is stressful. We propose that when examining the role of stress or pressure in cognitive performance it is important to consider the type of pressure, the stress response, and the aspect of cognition assessed. In Experiment 1, outcome pressure was not experienced as stressful but did lead to impaired performance on a rule-based (RB) category learning task and not a more procedural information-integration (II) task. In Experiment 2, the addition of monitoring pressure resulted in a modest stress response to combined pressure and impairment on both tasks. Across experiments, higher stress appraisals were associated with decreased performance on the RB, but not the II, task. In turn, higher stress-reactivity (heart rate) was associated with enhanced performance on the II, but not the RB, task. This work represents an initial step towards integrating the stress-cognition and pressure-cognition literatures and suggests that integrating these fields may require consideration of the type of pressure, the stress-response, and the cognitive system mediating performance.
\end{abstract}

Key words: Pressure, Stress, Category Learning, Cognition

\section{Introduction}

From family life to social life to work life, pressure and stress are so ubiquitous in modern life that it is no surprise that psychologists have taken great interest in the impact of pressure and stress on cognitive performance. Although pressure-cognition and stress-cognition research have proceeded somewhat independently, it is quite common to assume that pressure is stressful (Beilock \& DeCaro, 2007; Masters, 1992; Staal, 2004). Despite the face validity of this assumption, there have been few direct tests of this prediction. This question is particularly important in light of recent studies demonstrating that cognitive performance can vary as a function of the pressure manipulation (DeCaro et al., 2011) and the stress response (e.g., Ell et al., 2011). In the current research, we take an initial step towards integrating pressure and stress research by examining whether pressure is experienced as stressful.

\section{What is pressure?}

Individuals experience pressure when they must perform to their potential in order to achieve a goal (Baumeister, 1984). This type of outcome pressure is often induced by increasing the difficulty of reaching some goal and/or providing an incentive that is contingent on performance. Outcome pressure is thought to co-opt working memory and attentional resources, resulting in impairment in cognitive tasks dependent on these processes (Beilock \& Carr, 2005; Lewis \& Linder, 1997; Markman et al., 2006).

Pressure may also be induced by social evaluation or social monitoring (e.g., an evaluative other present and/or videotaping for later evaluation - DeCaro, et al., 2011; Gimmig et al., 2006). Monitoring pressure, in contrast, is more likely to encourage selfmonitoring of task performance than to co-opt working memory and attentional resources, resulting in impairment in cognitive tasks dependent on procedural knowledge (DeCaro, et al., 2011). Consistent with these 
predictions, outcome pressure has been shown to impair performance on cognitive tasks dependent on working memory, and monitoring pressure impairs performance on more procedural cognitive tasks (e.g. Decaro, et al., 2011). Importantly, however, many pressure situations are multifaceted including both aspects of outcome and monitoring pressure. Such combined pressure situations have been argued to negatively impact performance on both working-memory dependent and procedural-knowledge dependent tasks, although this prediction has yet to be tested (DeCaro, et al., 2011).

\section{What is Stress?}

As with pressure, stress is a multifaceted construct. Variability exists in individual responses to potential stressors (i.e. events, situations). Individuals experience more distress when the perceived demands of a situation exceed their resources to cope (e.g. Lazarus \& Folkman, 1994). Higher levels of distress are marked by the psychological experience of threat, and activation of both the sympathetic nervous system (SNS) and the hypothalamic pituitary adrenal axis (HPA) (Blascovich \& Tomaka, 1996; Dienstbier, 1989; Lazarus \& Folkman, 1984; Lupien et al., 2007; McEwen \& Sapolsky, 1995). When, however, individuals perceive adequate resources to cope with the demands of the situation, they may experience less distress (e.g., Lazarus \& Folkman, 1984).

Stressors that exhibit the most robust stress responses in the lab involve performance situations in which individuals are evaluated by others in a domain of personal importance, and in which they are motivated to do well (Blascovich \& Tomaka, 1996; Dickerson \& Kemeny, 2004). Such social-evaluative stressors share elements of both outcome pressure and monitoring pressure and, like pressure, have been shown to impair cognitive performance depending upon an individual's stress response (Ell, et al., 2011; Kassam et al., 2009; Payne et al., 2007). While a distress response is commonly associated with negative cognitive task performance, it may facilitate performance on more procedural cognitive tasks that are less dependent on working memory and attentional resources (e.g. Ell, et al., 2011). For example, distress has been shown to impair working memory and attentional control (e.g., Plessow et al., 2012; Schoofs et al., 2008) but also to bias processing toward procedural knowledge (Schwabe \& Wolf, 2012). These effects may be due in part to the "neuro-symphony" of neurotransmitters and stress steroids released in the stress response (e.g. norepinephrine and cortisol; Joels \& Baram, 2009). In contrast to pressure research in which it is the variability in the pressure situation (outcome, monitoring, combined) that is argued to be of critical import to understanding the consequences of pressure for cognition, the consequences of a stressor on cognition have been argued to depend critically on variability in the stress response.

\section{The Current Investigation: Is Pressure Stressful?}

Many manipulations intended to increase pressure, particularly monitoring pressure manipulations, include characteristics that might be expected to lead to a stress response. Indeed, the words pressure and stress are sometimes used interchangeably in the literature (e.g., Beilock \& DeCaro, 2007; DeCaro, et al., 2011; Staal, 2004). We propose that integrating the pressurecognition and stress-cognition literatures may require consideration of the type of pressure, the stress response, and the cognitive system mediating task performance.

As a model task, we focus on category learning (i.e. the process of establishing a memory trace that improves the efficiency of assigning novel objects to different groups). Category learning has attracted the interest of both pressure-cognition researchers (DeCaro, et al., 2011; Markman, et al., 2006; Worthy et al., 2009) and stress-cognition researchers (Ell, et al., 2011; Schwabe \& Wolf, 2012), making it a particularly useful paradigm given our goals. Moreover, there is extensive evidence suggesting that processing can be biased towards different cognitive systems by 
the categorization response) is assessed (Ashby \& Maddox, 2005). In particular, we focus on the rule-based (RB) and information-integration (II) tasks plotted in Figure 1. RB and II tasks are argued to be probes for different category learning systems that compete and vary in their dependence on working memory and attentional resources (with $\mathrm{RB}$ tasks being more dependent - Ashby et al., 1998; Ashby \& Ell, 2001; Ashby \& Maddox, 2005; but see Lewandowsky et al., 2012). For example, Markman and colleagues (Markman, et al., 2006) trained participants on the

Figure 1 tasks under low (i.e., participants were instructed to do their best) or high outcome pressure (i.e., participants were led to believe that their performance would determine if they, and a fictitious partner, would earn a monetary bonus). Consistent with the aforementioned research suggesting that outcome pressure co-opts working memory and attentional resources, and the assumption of competition between category learning systems, high outcome pressure impaired performance on the RB task and enhanced performance on the II task.

DeCaro and colleagues (DeCaro et al., 2011) extended this work and tested the hypothesis that the effect of pressure on performance in these cognitive tasks is also dependent on the type of pressure. Using a different set of RB and II tasks (i.e., stimuli varying along 4 , binary-valued dimensions), DeCaro and colleagues (2011) replicated the impairing effect of outcome pressure on an RB task, but observed no effect of outcome pressure on an II task. Monitoring pressure, in contrast, is argued to impair procedural tasks - tasks that are thought to be more sensitive to the effects of self-awareness and selfmonitoring. Consistent with this pressure-type hypothesis, DeCaro and colleagues found that monitoring pressure impaired performance on an II, and not an RB, task. Although combined pressure, or pressure that contains elements of both outcome and monitoring pressure, was not investigated, DeCaro and colleagues argued that combined

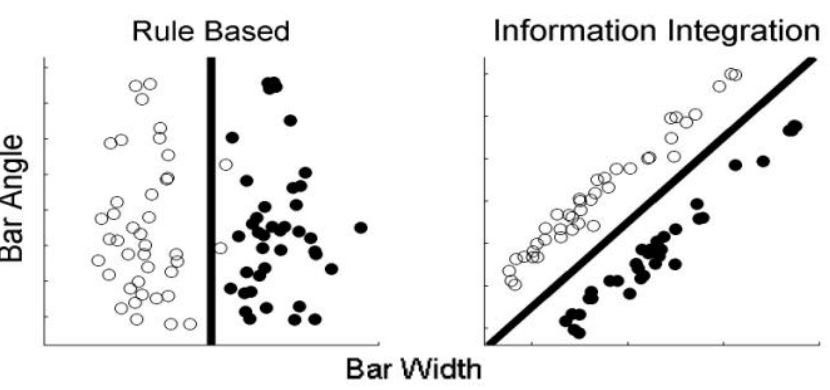

Figure 1. Scatterplots of the stimuli from the rule-based and information-integration tasks. The unfilled circles represent category A stimuli, the filled circles represent category B stimuli, and the solid lines are the optimal decision boundaries.

pressure would negatively impact performance in both RB and II tasks

Ell and colleagues (Ell et al. 2011) investigated the impact of a social-evaluative stressor on the subsequent performance in the RB and II tasks depicted in Figure 1. The social-evaluative stressor was adapted from the classic Trier Social Stress Test (TSST, Kirschbaum et al., 1993) which contains strong elements of both outcome and monitoring pressure (in addition to uncertainty and ego relevance elements). In contrast to the pressure manipulations described above, and in common with many investigations of the role of stress in cognition, this stressor occurred prior to the learning tasks (offline) and was not directly relevant to task performance yet participants' stress remained elevated into the learning period. Ell et al. (2011) found that increased distress enhanced performance on the II task and tended to decrease (although not significantly) performance on the RB task. Moreover, distress was associated with increased use of a task appropriate II decision strategy in the II task suggesting a bias away from rule-guided behavior.

Thus, RB and II categorization tasks have been studied in the context of both pressure and stress and have yielded mixed results, particularly for performance in II tasks. To begin to integrate the pressure-cognition and stress-cognition literatures in this area it is first necessary to answer the basic question of whether pressure is stressful. In two 
experiments, we test the basic question of whether pressure is experienced as stressful (Experiment 1, outcome pressure; Experiment 2, combined pressure), and the consequences of this pressure for performance in RB and II tasks.

\section{Experiment 1}

In Experiment 1, we replicated Markman et al. (2006) while recording markers of the stress response (i.e. distress appraisals, heart rate, blood pressure). We first examined whether the outcome pressure manipulation was experienced by participants as stressful. We then tested the hypothesis that outcome pressure would impair performance in the RB task and not impair performance on the II task (due to absence of monitoring pressure; DeCaro et al., 2011) or even enhance performance on the II task (due to competition; Markman, et al., 2006). Finally, a stress-variability perspective (Ell et al, 2011) would predict enhancement of II only to the extent that outcome pressure is distressing.

\section{Method}

\section{Participants and Design}

Undergraduates $(N=116)$ with normal $(20 / 20)$ or corrected to normal vision participated in a one hour session in exchange for course credit. Participants were randomly assigned to one of four experimental conditions (RB-low pressure $=$ 23; RB-high pressure $=36$; II-low pressure $=$ 20; II-high pressure $=35$ ). One participant was excluded from the RB-low pressure condition because they used only one response key throughout the experiment.

\section{Task and Procedure.}

All methods and procedures specific to the category learning tasks replicated Markman, et al. (2006) with one exception. As the impairing/enhancing effects of outcome pressure were evident across the initial 5 blocks in Markman et al., participants were trained on either the RB or II task for only 5, instead of 8 , blocks of 80 trials. The stimuli were sine-wave gratings weighted by a circular Gaussian filter that varied across trials in spatial frequency (cycles/degree of visual angle) and orientation (degrees of rotation counterclockwise from horizontal). On each trial, a single stimulus was presented and the participant was instructed to make a category assignment by pressing one of two response keys (labeled "A" or "B") with the index and middle fingers of their dominant hand (blood pressure measurements were taken from their non-dominant arm). There was no time limit for response and corrective feedback was provided immediately after each response (i.e., "Correct" or "Incorrect"). Two points were added to a point meter on the monitor following correct responses, but there was no change in points following incorrect responses. The stimuli were generated and presented using the Psychophysics Toolbox extensions (Brainard, 1997; Pelli, 1997) for MATLAB. The stimuli were displayed on a 17" LCD with $1280 \times 1024$ resolution in a dimly lit room.

Participants in the low outcome pressure condition were instructed to do their best. Participants in the high outcome pressure condition were instructed that they and a (fictional) partner would receive a monetary bonus if they both exceeded a performance criterion of 128 points (i.e., $80 \%$ correct) at the end of training and that their partner had met this criterion. Thus, earning the monetary bonus depended solely on the participant's performance. Participants were reminded of these instructions at the beginning of the final block. The performance criterion (128 points) was indicated by a line on the point meter.

\section{Distress Appraisal.}

Following the task, participants were asked to rate the extent to which they found the task stressful, demanding, effortful, and distressing on a 0 (not at all) to 6 (very much) scale. The responses were averaged to form a reliable index of distress $(\alpha=.82)$.

\section{Physical Reactivity.}

Upon arrival, sensors to monitor cardiovascular and hemodynamic reactivity were applied (ECG: electrocardiogram, BP: 
continual blood pressure). Participants then relaxed for a 5 min baseline. $\mathrm{HR}$ and MAP were recorded using BioPac MP150 hardware and BioPac Acquire software. Data were

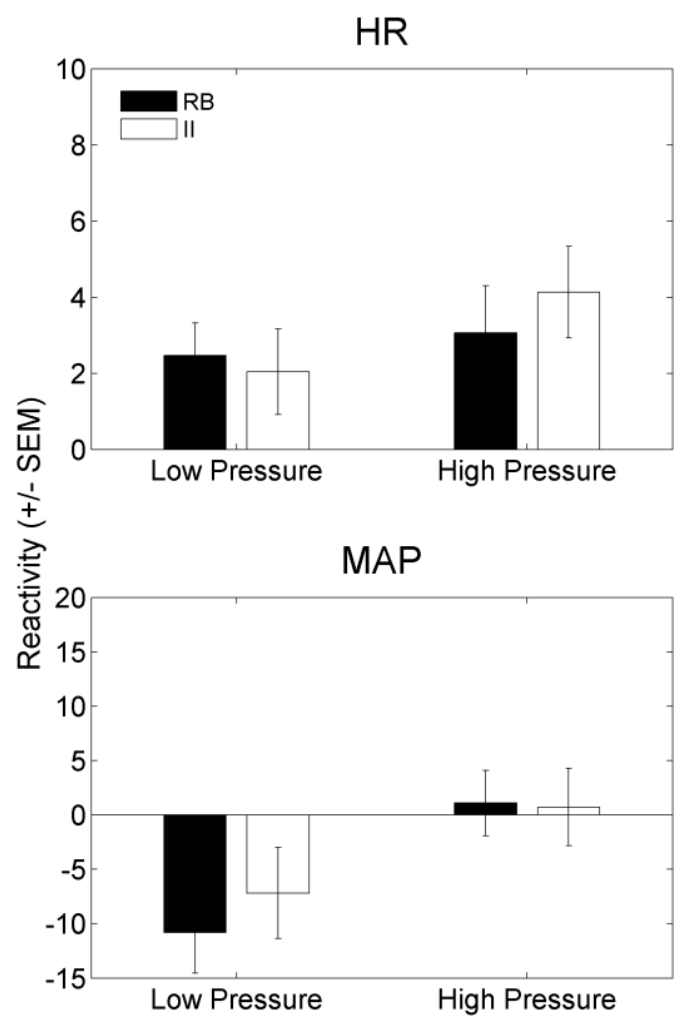

Figure 2. Average physiological reactivity at the beginning of the final block during Experiment 1 for $\mathrm{HR}$ (heart rate: beats/minute) and MAP (mean arterial pressure: $\mathrm{mm} \mathrm{Hg}$ ) as a function of the categorization task and pressure condition.

ensemble averaged over relevant minutes using Mindware software. We calculated average heart rate (HR, beats per minute) and mean arterial pressure [MAP in $\mathrm{mmHG}$, $\left.\frac{2 \text { (diastolic BP) }+ \text { systolic BP }}{3}\right]$ during baseline (last 2 min, most relaxed) and the final block of trials (first 2 min, highest pressure) ${ }^{1}$. As earning the

${ }^{1}$ We focus on the first 2 min of the final block to equate the time interval used for baseline measurements and because monetary bonus was contingent upon performance during the final block, pressure would be expected to be at its peak during the beginning of this block. Reactivity scores were computed by subtracting baseline from this peak level. Thus, positive/negative numbers indicate a rise/decline in HR or MAP.

\section{Results}

\section{Is Outcome Pressure Stressful?}

Distress Appraisals Participants did not find the outcome pressure particularly distressing as all participants reported levels well below the midpoint of the scale (High pressure: $M_{R B}$ $=2.10, S D_{R B}=1.26, M_{\| l}=1.26, S D_{\| l}=1.10$; Low pressure: $M_{R B}=2.07, S D_{R B}=.91, M_{\| l}=$ 1.26, $S D_{\|}=1.10$ ). Further, a 2(task) by 2(pressure) ANOVA revealed no effect of outcome pressure on distress $(F(1,106)=$ 2.62, $\left.p=.11, \eta_{p}^{2}=.02\right)$ nor an interaction, $F(1,106)=2.15, p=.12, \eta_{p}^{2}=.03$. There was an effect of task with participants rating the RB task slightly more distressing than the II task, $F(1,106)=4.18, p=.04, \eta_{p}^{2}=.04$.

Physiological Reactivity Consistent with the low levels of distress, participants did not evidence physical reactivity to outcome pressure (see Figure 2). Importantly, there were no significant differences by pressure condition in baseline HR (all $F s<1, p s>.40$ ) or baseline MAP (all $F s \leq 2.34$, ps $\geq .13$ ). No effects of pressure condition, task or the interaction were observed for $\mathrm{HR}$ reactivity (all $F s<1.20$, all $p s>.27$ ). Although there was a significant effect of pressure condition on MAP reactivity $F(1,86)=7.12, p<.05, \eta_{p}{ }^{2}=$ .08 ), this effect was not a result of increased MAP in the high pressure condition (see Figure 2). No other effects were observed for $\operatorname{MAP}(F s<.30, p s>.55)^{2}$.

cardiovascular responses recover relatively quickly from stress (Linden et al., 1997).

\footnotetext{
${ }^{2}$ Note that the degrees of freedom for our physiological measures fluctuate slightly as a result of missing or unscorable data due to equipment issues (RB-low pressure $=4$; RB-high pressure $=6$; II-low pressure $=2$; II-high pressure $=6$ ) .
} 
Does Outcome Pressure Impair Cognitive Performance?

Accuracy Analyses Consistent with predictions, outcome pressure significantly impaired accuracy in the RB task (see Figure 3). We examined the effect of pressure by block separately for each task condition (with block as the within-subjects factor, Greenhouse-Geisser corrected for violation of sphericity). As is evident in Figure 3, participants in all conditions improved in accuracy over blocks (RB block: $F(2.65,148.55)=39.92, p<.05, \eta_{p}{ }^{2}=.42 ;$ II block: $F(3.10,164.06)=30.98, \mathrm{p}<.05, \eta_{p}{ }^{2}=$ $.01)$.

Outcome pressure only negatively impacted performance in the RB task (RB pressure: $F(1,56)=5.96, p<.05, \eta_{p}{ }^{2}=.10$; II pressure: $F(1,53)=.69, p=.41, \eta_{p}^{2}=.01$ ) and this effect was consistent across blocks (RB block $x$ pressure: $F(2.65,148.55)=.65, p$ $=.57, \quad \eta_{p}^{2}=.01 ; \|$ block $\mathrm{x}$ pressure: $\left.F(3.10,164.06), p=.71, \eta_{p}{ }^{2}=.01\right)$. Consistent with a pressure-type perspective, (DeCaro et al., 2011) performance on the II task was not impaired. There was no evidence of enhancement of II performance in the high outcome pressure condition.

Model-Based Analyses Analysis of the accuracy data does not directly address the question of what decision strategies were used to perform the categorization tasks. For instance, does the impairment in the RB task reflect a shift to a less optimal decision strategy or an increase in guessing? The following analysis represents a quantitative approach to investigating these questions. Three different types of models were evaluated, each based on a different assumption concerning the participant's strategy. Rule-based models assume that the participant sets decision criteria on one (or both) stimulus dimensions (e.g., if the bars are wide, respond $A$; otherwise respond $B$ ). Information-integration models assume that the participant integrates the stimulus information from both dimensions prior to making a categorization decision. Finally, random responder models assume that the participant guessed. Each of these models was fit separately to the data from every response block for all participants using a standard maximum likelihood procedure for parameter estimation (Ashby, 1992b; Wickens, 1982) and the Bayes information

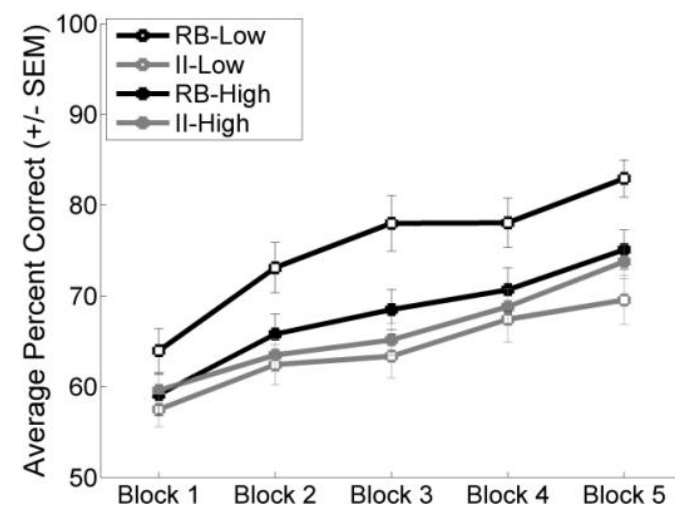

Figure 3. Average accuracy across blocks in Experiment 1 as a function of the categorization task and pressure condition.

criterion for goodness-of-fit (Schwarz, 1978) (see Appendix A for a more detailed description of the models and fitting procedure).

For brevity, we focus on the results from the final block (Table 1). In the RB task, there was a reduction in the dominance of $\mathrm{RB}$ strategies (and increase in guessing) in the high pressure condition, but this shift in the distribution of best-fitting models was not significant $\left[X^{2}(2)=2.49, \quad p=.29\right]$. Nevertheless, the increase in guessing likely contributed to the reduced accuracy in the RB-high pressure condition as the subset of participants best fit by RR models $(M=55.83$, $S D=4.42$ ) performed much worse than those best fit by RB models $(M=82.14, S D=6.85)$ or II models $(M=76.67, S D=6.88)$. In the II task, pressure had little effect on the distribution of best fitting strategies $\left[X^{2}(2)=\right.$ $.51, \mathrm{p}=.78]$. In sum, pressure was neither psychologically nor physiologically stressful, but consistent with previous work, pressure impaired performance on a RB task. 
Table 1. Proportion of participants best fit by each model type during the final block

$$
\text { Model Type }
$$

Condition
RB .82 .67 .30 .31 .31
.09

.08 .45 .51
RR .09 .25 .25 .17

II: High Outcome Pressure

\section{1}

Note. RB - rule based; II - information integration; RR - randd
High Pressure data do not sum to 1 due to rounding error.

\section{Discussion}

Our data suggest that the answer to the basic question of whether outcome pressure is stressful is: No. At the group level, participants did not find outcome pressure to be psychologically or physiologically distressing. Consistent with DeCaro and colleagues (2011), outcome pressure impaired performance in the RB task and did not impair performance in the II task. Collectively, these data are most consistent with the hypothesis that outcome pressure coopts working memory and attentional resources, leading to selective impairment in the RB task. It may be the case that higher levels of distress are required to evidence the enhancement of II performance demonstrated by Ell et al. (2011) Thus, in Experiment 2 we augment our outcome pressure with monitoring pressure in an effort to increase distress and to test the hypotheses for combined pressure set forth by DeCaro et al. (2011) (i.e. impairment in both II and RB tasks).

\section{Experiment 2}

In Experiment 2, we tested predictions regarding the consequences of combined pressure for stress reactivity and cognitive performance. To create our combined pressure condition, we augmented the outcome pressure manipulation from Markman et al. (2006) with monitoring pressure by adding elements of social evaluation. Social evaluation is a key component of many classic stress manipulations (see Dickerson \& Kemeny, 2004 for a review) and may lead to greater stress reactivity than outcome pressure alone. In addition we examined the theoretical predictions from DeCaro et al. (2011) that combined pressure would impair performance on both RB and II tasks.

\section{Method}

\section{Participants and Design}

Undergraduates ( $\mathrm{N}=103$; normal or corrected to normal vision) participating for course credit were randomly assigned to one of the four experimental conditions $(\mathrm{RB}$, low pressure $=$ 28; RB, high combined pressure $=26$; II, low pressure $=23$; II, high combined pressure $=$ 26). ${ }^{3}$

\footnotetext{
${ }^{3}$ Due to equipment and computer issues we have missing data for both the categorization task (II-low pressure $=1$; II-high
} 


\section{Task and Procedure}

The tasks and procedure were identical to Experiment 1 with the exception that participants in the high combined pressure condition experienced monitoring pressure in addition to outcome pressure. To subtly heighten social evaluation, participants engaged in a brief interaction over an intercom with the fictional partner. Following the task instructions, participants introduced

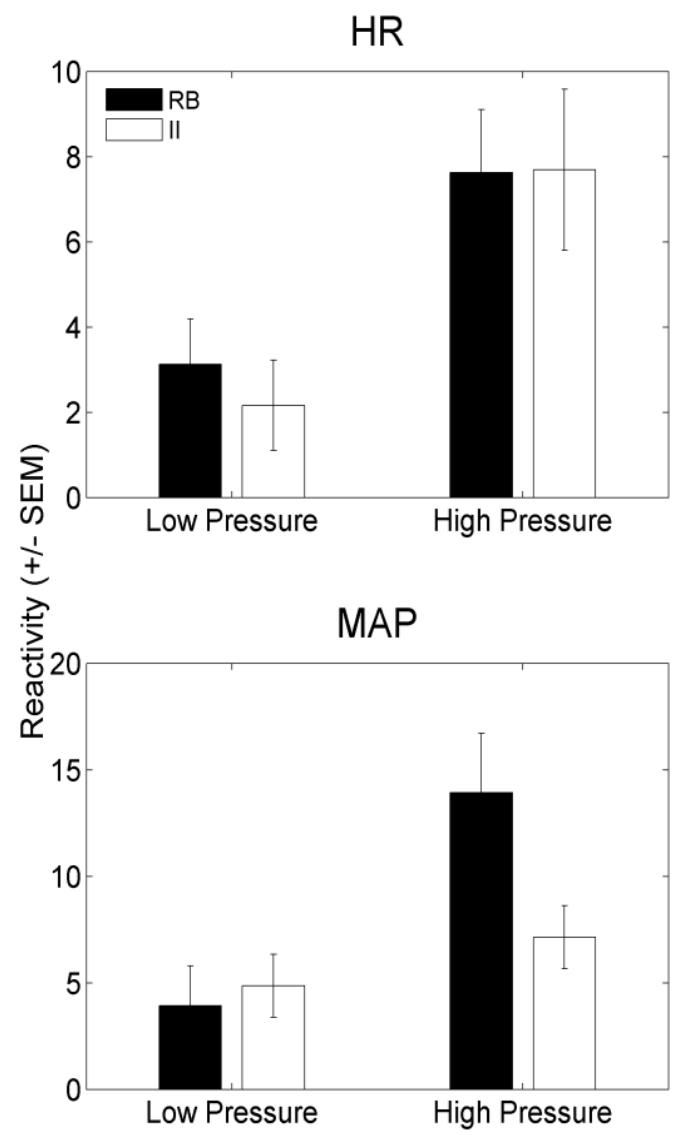

Figure 4. Average physiological reactivity at the beginning of the final block during Experiment 2 for HR (heart rate: beats/minute) and MAP (mean arterial pressure: $\mathrm{mm} \mathrm{Hg}$ ) as a function of the categorization task and pressure condition.

pressure $=1$ ), and the physiological markers (RB-low pressure $=1$; RB-high pressure $=1$; II-high pressure $=1$ ). themselves to their partner over an intercom. The partner then stated, "I did meet the criterion, so it is all up to you...good luck." The experimenter reinforced the social evaluation of the partner by stating, "Remember, you will have a chance to discuss your performance with your partner afterward" (see Appendix B for the full script). Participants in the low pressure condition were simply asked to do their best. Physical reactivity and distress appraisals $(\alpha=.78)$ were measured as described in Experiment 1.

Results

\section{Is Combined Pressure Stressful?}

Distress Appraisal While participants in the high combined pressure condition $\left(M_{\text {high }}=\right.$ 2.29, $S D_{\text {high }}=1.23$ ) reported significantly higher distress appraisals than participants in the low pressure condition $\left(M_{\text {low }}=1.71, S D_{\text {low }}\right.$ $\left.=1.23 ; F(1,98)=5.82, p<.05, \eta_{p}^{2}=.06\right)$, it should be noted that as in Experiment 1, distress remained below the midpoint of the scale. We observed no effect of task $(F(1,98)$ $\left.=1.23, p=.27, \eta_{p}^{2}=.01\right)$ nor of the interaction $\left(F(1,98)=3.34, p=.07, \eta_{p}^{2}=.03\right)$.

Physiological Reactivity Adding the subtle manipulation of monitoring pressure to the outcome pressure from Experiment 1 resulted in a modest, but significant, increase in HR and MAP relative to both baseline and the low pressure condition (see Figure 4). Importantly, no significant differences in MAP or HR were observed at baseline, although the effect of pressure condition approached significance for $\operatorname{HR}\left(F(1,85)=3.33, p=.07, \eta_{p}{ }^{2}=.04\right.$; all other $F s<1.54, p s>.21)$.

The addition of monitoring pressure to the outcome pressure used in Experiment 1 resulted in significant main effects of pressure for both HR reactivity $(F(1,85)=13.18, p<$ $\left..05, \eta_{p}^{2}=.13\right)$ and MAP reactivity $(F(1,84)=$ 9.34, $\left.p<.05, \eta_{p}^{2}=.10\right)$. Moreover, the modest increase in HR and MAP observed in the combined pressure conditions was significantly different from baseline $(0 ; \mathrm{HR}: t$ $(42)=6.34, p<.05, d=1.96$; MAP: $t(43)=$ $6.44, p<.05, d=1.96)$. While the interaction approached significance for $\operatorname{MAP}(F(1,84)=$ 
Table 2. Proportion of participants best fit by each model type during the final block

$$
\text { Model Type }
$$

Condition

$\begin{array}{lll}\text { RB } & \text { II }\end{array}$

RB: Low Pressure

.75

.11

.14

RB: High Combined Pressure

.62

.12

.27

II: Low Pressure

.45

.50

.05

II: High Combined Pressure

.40

.20

.40

Note. $R B$ - rule based; II - information integration; $R R$ - random responder. The

RB: Combined Pressure data do not sum to 1 due to rounding error.

3.69, $\left.p=.06, \eta_{p}^{2}=.04\right)$, no significant effect of task, or moderation by task, was observed ( $F S$ $<2.14$, ps > .14). Thus, adding a subtle manipulation of monitoring pressure to the outcome pressure from Experiment 1 resulted in a modest, but significant, increase in HR and MAP relative to both baseline and low pressure.

Does Outcome Pressure Impair Cognitive Performance?

Accuracy Analyses Consistent with predictions from DeCaro et al. (2011), combined pressure impaired performance on both the RB and II tasks (see Figure 5). As in Experiment 1, participants in all conditions improved their accuracy over blocks (RB: $F(2.88,149.73)=50.79, p<.05, \eta_{p}{ }^{2}=.49 ;$ II $\left.F(3.27,147.31)=24.02, p<.05, \eta_{p}{ }^{2}=.35\right)$. Participants under high combined pressure evidenced significantly lower performance in the $\operatorname{RB}\left(F(1,52)=4.84, p<.05, \eta_{p}^{2}=.09\right)$ and II tasks $\left(F(1,45)=8.36, p<.05, \eta_{p}{ }^{2}=.16\right)$ relative to the low pressure condition; and these effects were not moderated by block (RB: $F(2.88,149.73)=.59, p=.68, \eta_{p}^{2}=$ .009; II: $F(3.27,147.31)=1.08, p=.36, \eta_{p}{ }^{2}=$
$.02)$.

Model-Based Analyses In order to investigate any differences in the decision strategies used by participants, the models described in Experiment 1 were also fit to these data. For

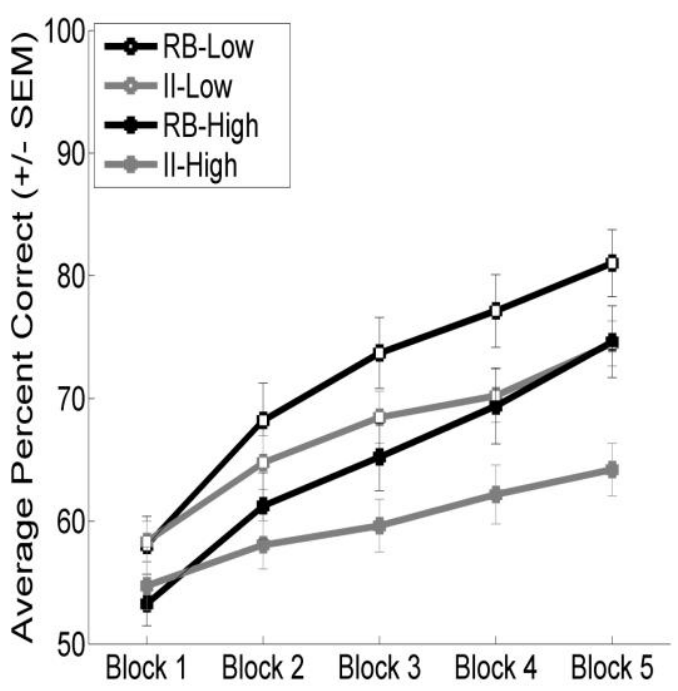

Figure 5. Average accuracy across blocks in Experiment 2 as a function of the categorization task and pressure condition. 
brevity, we focus on the results from the final block (Table 2). In both tasks, there was an increase in the dominance of guessing models in the combined pressure condition. Although the difference in the distribution of best fitting models between pressure conditions was statistically significant in only the II task [RB: $X^{2}(2)=1.42, p=.49 ;$ II: $x^{2}(2)$ $=9.46, p<.05]$, the increase in guessing likely contributed to the pressure impairment in both tasks as the subset of participants best fit by RR models $\left(M_{R B}\right.$ task $=52.68, S D_{R B}$ task $=3.78 ; M_{l l}$ task $=53.75, S D_{l l}$ task $\left.=5.59\right)$ performed much worse than those best fit by RB models $\left(M_{R B}\right.$ task $=81.48, S D_{R B}$ task $=8.96$ or II models $\left(M_{\| l}\right.$ task $=70.25, S D_{I I}$ task $\left.=3.47\right)$.

Is Distress Associated with Cognitive Performance at These More Modest Levels?

Previous work has demonstrated enhancement of performance in an II task with higher levels of distress (Ell, et al., 2011). As shown in Figure 6, the stress reactivity in the combined pressure condition of Experiment 2 is orders of magnitude lower than that observed in the social- evaluative stressor used by Ell et al. at peak stress (i.e., a modified version of the TSST; reactivity continued into the learning period in Ell et al.) 4

It is important to note, however, that although all participants faced the same social evaluative stressor in Ell et al., there was considerable individual variability in stress reactivity. The more distress participant's evidenced, the higher their II performance and the lower their RB performance (although the RB effects were not significant). Thus, while there may be mean differences in reactivity by pressure condition in Experiment 2, we would not expect the relationship between reactivity and cognitive performance to differ by pressure condition or Experiment. Accordingly, we

\footnotetext{
${ }^{4}$ As expected, physiological reactivity varied across the three experiments plotted in Figure 6 for both physiological variables [HR: $F(2,130)=47.65, p<.05, \eta_{p}{ }^{2}=.42$; MAP: $F(2,132)=$ 202.95, $\left.p<.05, \eta_{p}{ }^{2}=.26\right]$. In general, post hoc analyses (Student-Newman-Keuls) indicated that physiological reactivity was ordered from highest-to-lowest as described in Figure 6 $(p$ 's $<.05)$ with the one exception being the HR difference for the present experiments $(p=.06)$.
}

examined the relationships between distress (appraisals, HR reactivity, and MAP reactivity) and cognitive performance within each task condition collapsed across condition and experiment ${ }^{5}$.

While the level of distress in the current studies was more modest than observed in Ell et al., the more distress participants reported the lower their accuracy in the RB task $(r(110)$ $=-.47, p<.001)$ but not the II task $(r(100)=-$ $.15, p=.17)$. This impairment effect was replicated when examining the point biserial correlation between task appropriate strategy use (appropriate vs. inappropriate; see Appendix A) and reported distress. The more distress participants reported, the less likely participants were to use task appropriate rule based strategies in the RB task $(r(110)=-.42$, $p<.001)$. In contrast, distress did not predict appropriate use of II strategies in the II task, $r(100)=-.04, p=.67$. Consistent with the perspective that distress may enhance II performance, even at this modest level of distress higher heart rate reactivity was associated with better accuracy in the II task $(r(86)=.29, p<.01)$ but not the RB task $(r(91)$ $=.16, p=.13)$. This pattern of enhancement in the II task was also evident in the association between heart rate reactivity and the use of task appropriate strategies $\left(r_{l /}(86)=\right.$ $\left..25, p<.05 ; r_{R B}(91)=.02, p=.88\right)$. No effects were observed for blood pressure reactivity at these modest levels of distress (Accuracy: $r_{l /}(83)=.06, p=.57 ; r_{R B}(93)=.16, p=.11$; Appropriate Strategy Use: $r_{l /}(83)=.15, p=.17$; $\left.r_{R B}(93)=.02, p=.85\right)$.

\section{Discussion}

In Experiment 2, we added monitoring pressure to the outcome pressure manipulation used by Markman et al., 2006. The addition of social evaluation did indeed raise the intensity of the stress response.

\footnotetext{
${ }^{5}$ We first used moderated regression to examine whether the effects of distress on performance within each task were moderated by experiment and pressure condition. They were not. All relevant interaction terms [e.g. Distress Marker (centered at the mean) $X$ Task $(0=$ II) $X$ Experiment $(0=$ Experiment 1) $X$ Pressure Condition ( $0=$ low pressure)] were not significant using either accuracy or strategy use as an outcome variable and heart rate, appraisals, or blood pressure as the distress marker.
} 
While more modest than the stress response observed in Ell et al. (2011; see Figure 6), participants did perceive the combined pressure as more distressing and reported higher $\mathrm{HR}$ and MAP reactivity relative to the control condition. Consistent with the theorizing of DeCaro and colleagues (2011), combined pressure impaired both RB and II tasks relative to the control condition. The addition of monitoring pressure to the outcome manipulation from Experiment 1 led to an impairment of II performance that was not evident with outcome pressure alone. Model based analyses suggest that this impairment may be due to an increase in random responding in the II task under combined pressure.

We also found mixed evidence for the relationships between distress and task performance at these modest levels of stress reactivity. As hypothesized, accuracy and appropriate strategy use in RB tasks were impaired by distress - but only self-reported distress. In contrast, accuracy and appropriate strategy use in II tasks were enhanced by distress - but only as marked by heart rate reactivity.

\section{General Discussion}

The current research represents a modest first step toward integrating the pressurecognition and stress-cognition literatures. We believe the concepts of pressure, stress and cognition to be multifaceted. When examining the effects of a stressor on cognitive performance it is important to consider the type of pressure exerted (outcome, monitoring, or combined), stress reactivity and the cognitive system mediating task performance. As an initial step toward examining these broader questions, we first addressed the basic question of whether pressure is indeed stressful. Across two experiments, we examined the stress response to common pressure manipulations, and the consequences for categorization tasks thought to depend on different learning systems.

Is Pressure Stressful?

In Experiment 1, we found that outcome pressure was not experienced by participants as stressful. This is particularly important for future attempts at reconciling stress-cognition and pressure-cognition findings as the terms "stress" and "pressure" are often conflated in the literature. In Experiment 2, the addition of a very subtle manipulation of social evaluation increased monitoring pressure and resulted in significant, but modest, stress reactivity to this combined pressure. Participants reported significantly higher heart rate reactivity, blood pressure reactivity and distress appraisals in the combined pressure condition relative to the low pressure condition. As noted in Figure6, however, this stress response was substantially lower than that observed by Ell et al. (2011). As we work toward integrating the stress cognition and pressure cognition literatures, it may be important to distinguish when, and to what degree, pressure is experienced as distressing.

\section{Does Pressure Impair Cognitive Performance?}

Consistent with the findings from DeCaro et al. (2011) and Markman et al. (2006), outcome pressure and combined pressure impaired performance on the category learning task thought to be more dependent upon working memory and attentional resources (i.e., a RB task). These data are consistent with the hypothesis that outcome pressure serves as a distraction/divides attention, impairing performance on tasks dependent on working memory. Indeed, this may explain why participants tended to engage in more random responding in the RB task in the high pressure conditions relative to the no pressure conditions, although this effect was not significant in either study.

Our findings for the effects of pressure on II task performance were consistent with the 
theorizing and findings of DeCaro et al., 2011. Outcome pressure did not impair performance on a more procedural-based category learning task (i.e., an II task). Combined pressure, in contrast, led to impairments in accuracy and more random responding in the II task relative to the control condition, arguably due to increased self awareness resulting from monitoring (DeCaro et al., 2011).

We did not find that outcome pressure enhanced II performance, contrary to the findings of Markman et al (2006). As the prominent theoretical perspective on the learning of RB and II tasks (Ashby, et al., 1998) does not distinguish between types of pressure, the consequences of adding monitoring pressure (combined pressure) to the predictions of Markman et al are unclear. If adding monitoring pressure is perceived as simply increasing the intensity of the pressure, perhaps enhancement on the II task might have been expected. There is no easy explanation for our failure to replicate the enhancement observed by Markman et al. Perhaps the participants in Markman et al. found the outcome pressure more distressing than our participants (i.e. higher levels of distress may be associated with enhancement of II; Ell et. al., 2011).

Distress and Cognitive Performance: More Questions than Answers?

How do the current findings relate to those observed by Ell and colleagues (2011)? Ell and colleagues found that distress enhanced performance on an II task. For both accuracy and appropriate strategy use, the higher participants distress (i.e. appraisals, total peripheral resistance, cardiac output) the better their II performance. We did not observe enhanced II performance in the only condition that evidenced significant stress reactivity above baseline: combined pressure. Yet, from the stress-variability perspective of Ell et al., individuals vary in their response to pressure and it is those that respond with higher levels of distress (regardless of pressure condition) that may evidence enhanced II performance.
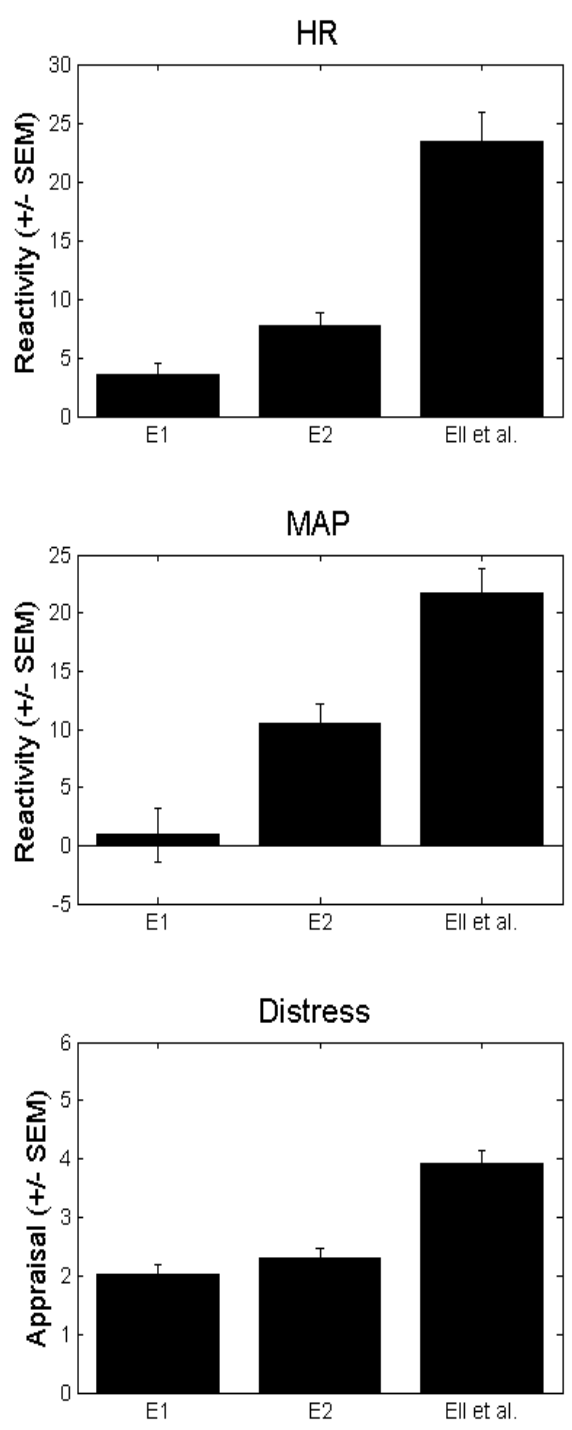

Figure 6. Average physiological reactivity and distress appraisals from the high pressure conditions (averaged across tasks) of Experiment 1 (E1), Experiment 2 (E2) and the final two min. of a modified version of the TSST that was administered in Ell et al. (2011). HR (heart rate: beats per minute) and MAP (mean arterial pressure: $\mathrm{mm} \mathrm{Hg}$ ).

Accordingly, we analyzed the associations between our markers of stress reactivity and task performance collapsed across condition and experiment. Our results were mixed. Consistent with the findings that Ell et al. observed with different distress markers, 
higher levels of heart rate reactivity were associated with greater accuracy, as well as, more appropriate strategy use in the II task but not in the RB task. It is particularly intriguing to observe this association at these lower levels of stress reactivity when behavior is often unassociated with reactivity (Lupien, et al., 2007; Roozendaal, 2002). Indeed, II and RB task performance were unassociated with blood pressure reactivity. In contrast to the physical distress variable predictive of II performance, RB performance was related to the self-report marker of distress. The more participants reported feeling that the task was stressful, effortful, demanding and distressing the lower their accuracy and appropriate strategy use in the RB task. Self-reported distress was unrelated to performance in the Il task.

Reconciling the current mixed findings with Ell et al is further complicated by differences in the levels of participants' stress reactivity. While distress rose with the addition of monitoring pressure in Experiment 2 , as can be seen in Figure 6 distress reactivity was more modest than that observed in Ell et al. In addition to aspects of outcome and monitoring pressure the TSST incorporates a number of other factors that are associated with a more robust stress response (e.g., uncontrollability - Dickerson \& Kemeny, 2004). Further the TSST is a 20 min social evaluative stressor developed to activate the Hypothalimic Pituitary Adrenal Axis (HPA) and measurable increases in cortisol (Kirschbaum, et al., 1993). Although combined pressure resulted in significant increases for both MAP and HR (relative to baseline), these effects were relatively modest when compared to those observed with the TSST. Thus, it may be that consistent associations between stress reactivity and task performance are more evident with higher levels of reactivity.

Yet, while the mixed findings between distress reactivity and performance could be due to the lower intensity of the response, there are other important factors to consider. Our analyses assume a linear relationship between distress and cognitive performance. It is conceivable that this relationship could vary across levels of distress. Certainly the "neuro-symphony" (Joels \& Baram, 2009) of the stress response is likely to vary (e.g. cortisol more evident at higher levels of intensity). A broader representation of data across the continuum of the stress response would help clarify whether the relationship between distress and cognitive performance in the RB and II tasks is non-linear.

In addition, there is considerable methodological diversity in the way in which pressure has been implemented in the laboratory setting. Some researchers have simply used time pressure or performance bonuses whereas others have incorporated a social-evaluative component (see Staal, 2004 for a review). In addition, pressure manipulations are typically administered online (i.e., the pressure manipulation and task are concurrent) and often the manipulation is directly relevant to the task at hand (i.e., partner's bonus contingent on participant's performance). This stands in contrast to the majority of stress-cognition research that uses offline stressors (i.e., the stressor precedes the task but reactivity may remain high during the task as in Ell et al., 2011), stressors unrelated to the cognitive task (e.g., cold pressor; Smeets et al., 2008), and/or more intense prolonged stressors known to activate a cortisol response (i.e. TSST). These differences in method may stem from differences in the proposed mediators of the role of pressure and stress in cognitive performance. Much of the pressure literature posits cognitive explanations while in contrast much of the stress literature posits more biological ones. Our findings may raise more questions than answers - but they are important questions for future work integrating the stress cognition and pressure cognition literatures. Our data suggest that research examining factors related to the intensity, task relevance, type (outcome, monitoring, combined), and timing (online vs. offline) of stressors/pressure manipulations is needed.

\section{Conclusions}

The goal of this research was to take an initial modest step towards integrating the pressure- 
cognition and stress-cognition literatures. While the terms pressure and stress are often used interchangeably in the literature, our data suggest that there are important distinctions: Pressure is not always stressful. Our findings regarding task performance support the hypotheses of DeCaro et al. demonstrating that differentiating pressure type may be important for understanding the relationship between pressure and performance in cognitive tasks dependent on different learning systems. Outcome pressure is assumed to impact cognitive performance by co-opting working memory and attentional resources thereby impairing performance on tasks dependent upon executive control processes. Monitoring pressure, in contrast, is assumed to increase awareness of the subcomponents of tasks thereby impairing performance on more procedural tasks. In our experiments, outcome pressure selectively impaired performance on the RB task, while the addition of monitoring pressure led to impairment in both RB and II tasks. This latter finding is novel and contributes to our understanding of the role of combined pressure in cognitive performance. These data also contribute to the growing appreciation in both the stress-cognition and pressure-cognition literatures of distinguishing the cognitive system mediating task performance when considering the consequences of pressure or stress. While our findings for the role of distress in cognitive performance were less clear at the low levels of reactivity observed, including the measurement of the physiological stress response in future studies of the pressurecognition relationship will be critical in elucidating this relationship given the possible interplay between stress-response variability and the cognitive systems mediating task performance.

\section{Appendix A: Model-Based Analyses}

To get a more detailed description of how participants categorized the stimuli, a number of different decision bound models (Ashby, 1992a; Maddox \& Ashby, 1993) were fit separately to the data for each participant from every block. Decision bound models are derived from general recognition theory (Ashby \& Townsend, 1986), a multivariate generalization of signal detection theory (Green \& Swets, 1966). It is assumed that, on each trial, the percept can be represented as a point in a multidimensional psychological space and that each participant constructs a decision bound to partition the perceptual space into response regions. The participant determines which region the percept is in, and then makes the corresponding response. While this decision strategy is deterministic, decision bound models predict probabilistic responding because of trial-by-trial perceptual and criterial noise (Ashby \& Lee, 1993).

The appendix briefly describes the decision bound models. For more details, see Ashby (1992a) or Maddox and Ashby (1993). The classification of these models as either rule-based or information-integration models is designed to reflect current theories of how these strategies are learned (e.g., Ashby, et al., 1998) and has received considerable empirical support (see Ashby \& Maddox, 2005; Maddox \& Ashby, 2004 for reviews).

\section{Rule-Based Models}

Unidimensional Classifier (UC) This model assumes that the stimulus space is partitioned into two regions by setting a criterion on one of the stimulus dimensions. Two versions of the UC were fit to these data. One version assumes that participants attended selectively to spatial frequency and the other version assumes participants attended selectively to orientation. The UC has two free parameters, one corresponds to the decision criterion on the attended dimension and the other corresponds to the variance of internal (perceptual and criterial) noise $\left(\sigma^{2}\right)$. A special case of the UC, the Optimal Unidimensional Classifier, assumes that participants use the unidimensional decision bound that maximizes accuracy. This special case has one free parameter $\left(\sigma^{2}\right)$

Conjunctive Classifier (CC) An alternative rule-based strategy is a conjunction rule involving separate decisions about the stimulus value on the two dimensions with the response assignment based on the outcome 
of these two decisions (Ashby \& Gott, 1988). The CC assumes that the participant partitions the stimulus space into four regions. Based on an initial inspection of the data, two versions of the $\mathrm{CC}$ were fit to these data. One version assumes that individuals assigned a stimulus to category $B$ if it was high on spatial frequency and low on orientation (i.e., the bars are thin and shallow); otherwise the stimulus would be assigned to category $A$. The other version assumes that individuals assigned a stimulus to category $A$ if it was low on spatial frequency and high on orientation (i.e., the bars are thick and steep); otherwise the stimulus would be assigned to category $B$. The CC has three free parameters: the decision criteria on the two dimensions and a common value of $\sigma^{2}$ for the two dimensions.

Information-Integration Models

The Linear Classifier (LC) This model assumes that a linear decision bound partitions the stimulus space into two regions. The LC differs from the CC in that the LC does not assume decisional selectiveattention (Ashby \& Townsend, 1986). This produces an information-integration decision strategy because it requires linear integration of the perceived values on the stimulus dimensions. The LC has three parameters, slope and intercept of the linear bound, and $\sigma^{2}$.

The Minimum Distance Classifier (MDC). This model assumes that there are a number of units representing a low-resolution map of the stimulus space (Ashby \& Waldron, 1999; Ashby et al., 2001; Maddox et al., 2004). On each trial, the participant determines which unit is closest to the perceived stimulus and produces the associated response. The version of the MDC tested here assumes two units because the category structures were generated from two multivariate normal distributions. Because the location of one of the units can be fixed, and because a uniform expansion or contraction of the space will not affect the location of the minimum-distance decision bounds, the MDC has four free parameters (three determining the location of the units and $\sigma^{2}$ ).

\section{Random Responder Models}

Equal Response Frequency (ERF) This model assumes that participants randomly assign stimuli to the two response frequencies in a manner that preserves the category base rates (i.e., $50 \%$ of the stimuli in each category). This model has no free parameters.

Biased Response Frequency (BRF) This model assumes that participants randomly assign stimuli to the two response frequencies in a manner that matches the participant's categorization response frequencies (i.e., the percentage of stimuli in each category is computed from the observed response frequencies). This model has no free parameters. Although the ERF and BRF are assumed to be consistent with guessing, these models would also likely provide the best account of participants that frequently shift to very different strategies.

\section{Model Fitting}

The model parameters were estimated using maximum likelihood (Ashby, 1992b; Wickens, 1982) and the goodness-of-fit statistic was

$$
\mathrm{BIC}=r \ln N-2 \ln L,
$$

where $N$ is the sample size, $r$ is the number of free parameters, and $L$ is the likelihood of the model given the data (Schwarz, 1978). The BIC statistic penalizes a model for poor fit and for extra free parameters. To find the best model among a set of competitors, one simply computes a BIC value for each model, and then chooses the model with the smallest BIC. For data analysis purposes, the bestfitting models were classified as being task appropriate or not. For the RB task, the UC models were task appropriate. For the II task, the LC and MDC were task appropriate.

\section{Appendix B: Experiment 2 Script}

The participant was informed of the name of their fictitious partner just prior to interacting with the fictitious partner via an intercom system. The fictitious partner (Jen or John) and the participant were matched on gender. Below is an example for a female participant. 
Experimenter (speaking into the intercom): Ok, Jen go ahead and introduce yourself to your partner.

Fictitious Partner (recording played over the intercom): Hi my name is Jen, nice to meet you.

Experimenter (speaking to the participant): Now go ahead and introduce yourself to Jen.

... After the participant responds...

Experimenter (speaking into the intercom): Jen, do you have anything you would like to tell your partner about the task?

Fictitious Partner (recording played over the intercom): Umm...no not really. Except...I did meet the criterion, so it is all up to you...good luck.

Experimenter (speaking to the participant): Great. Now that you have been introduced, let's get started. Remember, you will have a chance to discuss your performance with your partner afterward. The computer will reiterate many of the directions I have discussed with you, so if you have any questions feel free to speak up. Also, the computer will remind you of your partner's performance throughout the task, so when you get to the final block you will know how well you need to perform.

\section{Author Notes}

S. McCoy and S. Ell contributed equally to this research and first authorship was decided by the spin of a rigged roulette wheel. The authors would like to thank Todd Maddox, Art Markman, and Darrell Worthy for sharing the software for running the experiment as well as Ellen Newell and Joseph Wellman for assistance with data collection. Thanks to Dr. Barch and two anonymous reviewers for their helpful comments on an earlier draft of the manuscript. Correspondence concerning this article should be addressed to Shannon K. McCoy or Shawn W. Ell, Psychology Department, University of Maine, 5742 Little Hall, Room 301, Orono, ME 04469-5742 (email: shannon.mccoy@umit.maine.edu or shawn.ell@umit.maine.edu).

\section{References}

Ashby, F. G. (1992a). Multidimensional models of categorization. In F. G. Ashby (Ed.), Multidimensional models of perception and cognition. Hillsdale, NJ: Erlbaum.

Ashby, F. G. (1992b). Multivariate probability distributions. In F. G. Ashby (Ed.), Multidimensional models of perception and cognition (pp. 1-34). Hillsdale: Lawrence Erlbaum Associates, Inc.

Ashby, F. G., Alfonso-Reese, L. A., Turken, A. U., \& Waldron, E. M. (1998). A neuropsychological theory of multiple systems in category learning. Psychological Review, 105, 442-481.

Ashby, F. G., \& Ell, S. W. (2001). The neurobiology of human category learning. Trends in Cognitive Science, 5(5), 204210.

Ashby, F. G., \& Gott, R. E. (1988). Decision rules in the perception and categorization of multidimensional stimuli. Journal of Experimental Psychology: Learning, Memory, and Cognition, 14, 33-53.

Ashby, F. G., \& Lee, W. W. (1993). Perceptual variability as a fundamental axiom of perceptual science. In S. C. Masin (Ed.), Foundations of percpetual theory (pp. 369-399). Amsterdam: Elsevier.

Ashby, F. G., \& Maddox, W. T. (2005). Human category learning. Annual Review of Psychology, 56, 149-178. doi: 10.1146/annurev.psych.56.091103.07021 7

Ashby, F. G., \& Townsend, J. T. (1986). Varieties of perceptual independence. Psychological Review, 93, 154-179.

Ashby, F. G., \& Waldron, E. M. (1999). The nature of implicit categorization. Psychonomic Bulletin \& Review, 6, 363-378.

Ashby, F. G., Waldron, E. M., Lee, W. W., \& Berkman, A. (2001). Suboptimality in human categorization and identification. Journal of Experimental Psychology: General, 130(77-96).

Baumeister, R. F. (1984). Choking under preessure: Self-consciousness and paradoxical effects of incentives on skillful performance. Journal of Personality and Social Psychology, 46(610-620).

Beilock, S. L., \& Carr, T. H. (2005). When highpowered people fail: Working memory and "choking under pressure" in math. Psychological Science, 16, 101-105. 
Beilock, S. L., \& DeCaro, M. S. (2007). From poor performance to success under stress: Working memory, strategy selection, and mathematical problem solving under pressure. Journal of Experimental Psychology: Learning, Memory, and Cognition, 33, 983-998.

Blascovich, J., \& Tomaka, J. (1996). The biopsychosocial model of arousal regulation. Advances in Experimental Social Psychology, 28, 1-51.

Brainard, D. H. (1997). Psychophysics software for use with MATLAB. Spatial Vision, 10, 433436.

DeCaro, M. S., Thomas, R. D., Albert, N. B., \& Beilock, S. L. (2011). Choking under pressure: Multiple routes to skill failure. Journal of Experimental Psychology: General, 140, 390-406. doi: 10.1037/a0023466

Dickerson, S. S., \& Kemeny, M. E. (2004). Acute stressors and cortisol responses: A theoretical integration and synthesis of laboratory research. Psychological Bulletin, 130, 355-391.

Dienstbier, R. A. (1989). Arousal and physiological toughness: Implications for mental and physical health. Psychological Review, 96, 84-100.

Ell, S. W., Cosley, B., \& McCoy, S. K. (2011). When bad stress goes good: increased threat reactivity predicts improved category learning performance. Psychon Bull Rev, 18(1), 96-102. doi: 10.3758/s13423-010-0018-0

Gimmig, D., Huguet, P., \& Caverni, J. P. (2006). Choking under pressure and working memory capacity: Whem performance pressure reduces fluid intelligence. Psychonomic Bulletin \& Review, 13, 10051010.

Green, D. M., \& Swets, J. A. (1966). Signal detection theory and psychophysics. New York: Wiley.

Joels, M., \& Baram, T. Z. (2009). The neurosymphony of stress. Nature Reviews Neuroscience, 10(6), 459-466. doi: 10.1038/nrn2632

Kassam, K. S., Koslov, K., \& Mendes, W. B. (2009). Decisions under distress: Stress profiles influence anchoring and adjustment. Psychological Science, 20, 1394-1399.

Kirschbaum, C., Pirke, K. M., \& Helhammer, D. H. (1993). The "Trier Social Stress Test": A tool for investigating psychobiological stress responses in a laboratory setting. Neuropsychobiology, 28, 76-81.

Lazarus, R. S., \& Folkman, S. (1984). Stress, Appraisal, and Coping. New York: Springer.

Lewandowsky, S., Yang, L. X., Newell, B. R., \& Kalish, M. L. (2012). Working memory does not dissociate between different perceptual categorization tasks. J Exp Psychol Learn Mem Cogn, 38(4), 881-904. doi: $10.1037 / a 0027298$

Lewis, B., \& Linder, D. (1997). Thinking about choking? Attentional processes and paradoxical performance. Personality and Social Psychology Bulletin, 23, 937-944.

Linden, W., Earle, T. L., Gerin, W., \& Christenfeld, N. (1997). Physiological stress reactivity and recovery: Conceptual siblings separated at birth. Journal of Psychosomatic Research, 42, 117-135.

Lupien, S. J., Maheu, F., Tu, M., Fiocco, A., \& Schramek, T. E. (2007). The effects of stress and stress hormones on human cognition: Implications for the field of brain and cognition. Brain and Cognition, 65, 209-237.

Maddox, W. T., \& Ashby, F. G. (1993). Comparing decision bound and exemplar models of categorization. Perception Psychophysics, 53, 49-70.

Maddox, W. T., \& Ashby, F. G. (2004). Dissociating explicit and procedurallearning based systems of perceptual category learning. Behavioral Processes, 66, 309-332.

Maddox, W. T., Filoteo, J. V., Hejl, K. D., \& Ing, A. D. (2004). Category Number Impacts Rule-Based but not Information-Integration Category Learning: Further Evidence for Dissociable Category Learning Systems. Journal of Experimental Psychology: Learning, Memory, and Cognition, 30, 227-235.

Markman, A. B., Maddox, W. T., \& Worthy, D. A. (2006). Choking and excelling under pressure. Psychological Science, 17(11), 944-948. doi: 10.1111/j.14679280.2006.01809.x

Masters, R. S. W. (1992). Knowledge, knerves and know-how: The role of explicit versus implicit knowledge in the breakdown of a complex motor skill under pressure. British Journal of Psychology, 83, 343-358.

McEwen, B. S., \& Sapolsky, R. M. (1995). Stress and cognitive function. Current Opinion in Neurobiology, 5, 205-216. 
Payne, J. D., Jackson, E. D., Hoscheidt, S., Ryan, L., Jacobs, W. J., \& Nadel, L. (2007). Stress administered prior to encoding impairs neutral but enhances emotional long-term episodic memories. Learning \& Memory, 14, 861-868.

Pelli, D. G. (1997). The VideoToolbox software for visual psychophysics: Transforming numbers into movies. Spatial Vision, 10, 437-442.

Plessow, F., Schade, S., Kirschbaum, C., \& Fischer, R. (2012). Better not to deal with two tasks at the same time when stressed? Acute psychosocial stress reduces task shielding in dual-task performance. Cognitive, Affective, \& Behavioral Neuroscience, 12(3), 557-570. doi: 10.3758/s13415-012-0098-6

Roozendaal, B. (2002). Stress and memory: opposing effects of glucocorticoids on memory consolidation and memory retrieval. Neurobiology of Learning and Memory, 78(3), 578-595. doi: 10.1006/nlm3.2002.4080

Schoofs, D., Preub, D., \& Wolf, O. T. (2008). Psychosocial stress induces working memory impairments in an n-back paradigm. Psychoneuroendocrinology, 33, 643-653.

Schwabe, L., \& Wolf, O. T. (2012). Stress modulates the engagement of multiple memory systems in classification learning Journal of Neuroscience, 32(32), 1104211049. doi: 10.1523/JNEUROSCI.148412.2012

Schwarz, G. (1978). Estimating the dimension of a model. The Annals of Statistics, 6(2), 461464.

Smeets, T., Otgaar, H., Candel, I., \& Wolf, O. T. (2008). True or false? Memory is differentially affected by stress-induced cortisol elevations and sympathetic activity at consolidation and retrieval. Psychoneuroendocrinology, 33(10), 13781386.

doi: 10.1016/j.psyneuen.2008.07.009

Staal, M. A. (2004). Stress, cognition, and human performace: A literature review and conceptual framework. NASA TM 2004212824. Retrieved from from humanfactors.arc.nasa.gov/flightcognition/Public ations/IH_054_Staal.pdf.

Wickens, T. D. (1982). Models for behavior: Stochastic processes in psychology. San Francisco: W. H. Freeman.

Worthy, D. A., Markman, A. B., \& Maddox, W. T. (2009). Choking and excelling under pressure in experienced classifiers. Atten Percept Psychophys, 71(4), 924-935. doi: 10.3758/APP.71.4.924 
